\title{
AXIOMS FOR THE NORM RESIDUE ISOMORPHISM
}

\author{
C. WEIBEL
}

\begin{abstract}
We give an axiomatic framework for proving that the norm residue map is an isomorphism (i.e., for settling the motivic Bloch-Kato conjecture). This framework is a part of the Voevodsky-Rost program.
\end{abstract}

\section{INTRODUCTION}

The purpose of this paper is to give an axiomatic framework for proving (by induction on $n)$ that the norm residue map $K_{n}^{M}(k) / \ell \longrightarrow H_{\text {ét }}^{n}\left(k, \mu_{\ell}^{\otimes n}\right)$ is an isomorphism, for any prime $\ell>2$ and for any field $k$ such that $\ell$ is invertible in $k$.

Fix $\ell$ and $n$. By a Rost variety for a sequence $\underline{a}=\left(a_{1}, \ldots, a_{n}\right)$ of units in $k$, we shall mean a smooth projective variety $X$ of dimension $d=\ell^{n-1}-1$ satisfying the conditions of $[\mathrm{MC} / 1,6.3]$ or $[4,(0.1)]$; the exact definition is given in Definition 1.1 below. One key requirement is that $\left\{a_{1}, \ldots, a_{n}\right\}$ vanishes in $K_{n}^{M}(k(X)) / \ell$. Rost constructed such a variety in his 1998 preprint [1]; the proof that it satisfies these properties was published in [4].

main Theorem 0.1. Let $n$ and $\ell$ be such that the norm residues maps are isomorphisms for all $i<n$. Suppose that for every sequence $\underline{a}$ there is a direct summand $M$ of a Rost variety $X$, satisfying the Axioms given in 0.3 below.

Then the norm residue $\operatorname{map} K_{n}^{M}(k) / \ell \rightarrow H_{e ̂ t}^{n}\left(k, \mu_{\ell}^{\otimes n}\right)$ is an isomorphism.

To state the axioms, let $\mathfrak{X}$ denote the simplicial Čech scheme $p \mapsto X^{p+1}$; see $[\mathrm{MC} / 2,9.1]$. By abuse of notation, we will regard $\mathfrak{X}$ as a chain complex, and hence as an element of $\mathbf{D M}^{\text {eff }}$. We set $b=d /(\ell-1)=1+\ell+\cdots+\ell^{n-2}$.

Xdual Definition 0.2. There is a duality isomorphism $X^{*} \otimes \mathbb{L}^{d} \cong X$, where $X^{*}$ is the dual Chow motive of $X$ and $\mathbb{L}$ is the Lefschetz motive $\mathbb{Z}(1)$ [2]; see [MVW, 20.11]. By the $\mathfrak{X}$-dual $D p$ of the structure map $X \stackrel{p}{\longrightarrow} \mathbb{Z}$ we will mean the map

$$
\mathfrak{X} \otimes \mathbb{L}^{d} \rightarrow \mathbb{L}^{d} \stackrel{p^{*} \otimes \mathbb{L}^{d}}{\longrightarrow} X^{*} \otimes \mathbb{L}^{d} \cong X .
$$

Any summand $M$ of $X$ has a $\mathfrak{X}$-dual $\mathfrak{X} \otimes \mathbb{L}^{d} \stackrel{D y}{\longrightarrow} M$; see Remark 0.6.

axiom Axioms 0.3. (a) $M$ is a direct summand of $X$.

(b) The evident duality map $M^{*} \otimes \mathbb{L}^{d} \longrightarrow M$ is an isomorphism.

(c) There is a motive $D$, related to $y$ and $D y$ by two distinguished triangles:

$$
\begin{gathered}
D \otimes \mathbb{L}^{b} \rightarrow M \stackrel{y}{\longrightarrow} \mathfrak{X} \rightarrow, \\
\mathfrak{X} \otimes \mathbb{L}^{d} \stackrel{D y}{\longrightarrow} M \rightarrow D \rightarrow .
\end{gathered}
$$

Given (0.4), triangle (0.5) is equivalent to the duality assertion that $D^{*} \otimes \mathbb{L}^{d-b} \cong D$.

Date: December 18, 2006.

Weibel's research was supported by NSA grant MSPF-04G-184, and by the Oswald Veblen Fund. 
Mt Remark 0.6. The summand $M^{\prime}=M^{*} \otimes \mathbb{L}^{d}$ of $X$ is a priori different from $M$. Axiom $0.3(\mathrm{~b})$ says that $M^{\prime} \rightarrow X \rightarrow M$ is an isomorphism.

In the language of Chow motives, any direct summand $M=(X, e)$ of $X$ has a dual motive $M^{*}=\left(X, e^{t}, d\right)$ and a transpose summand $M^{\prime}=\left(X, e^{t}\right)$ of $X$. The sign differences between these languages is due to the fact that the category of Chow motives embeds contravariantly into $\mathbf{D} \mathbf{M}^{\mathrm{eff}}$.

In the language of $\mathbf{D} \mathbf{M}^{\text {eff }}$, the $\mathfrak{X}$-dual of the structure map $y: M \rightarrow \mathbb{Z}$ is the composition:

$$
\mathfrak{X} \otimes \mathbb{L}^{d} \rightarrow \mathbb{L}^{d} \stackrel{p^{*}}{\longrightarrow} X^{*} \otimes \mathbb{L}^{d} \rightarrow M^{*} \otimes \mathbb{L}^{d} \cong M^{\prime}
$$

Axiom $0.3(\mathrm{~b})$, allows us to identify the $\mathfrak{X}$-duals of $y$ and $y^{\prime}$.

This paper is an attempt to clarify the ending of the Voevodsky-Rost program to prove that the norm residue map is an isomorphism, and specifically the proof as presented in Voevodsky's 2003 preprint [MC/1]. One important feature of our Theorem 0.1 is that only published results (and [1]) are used.

When $\ell=2$, triangle $(0.4)$ was constructed in $[\mathrm{MC} / 2,4.4]$ using $D=\mathfrak{X}$, and $(0.5)$ is its $\mathfrak{X}$-dual. Axioms $0.3(\mathrm{a}-\mathrm{b})$ hold by a result of Rost (cited as [MC/2, 4.3].

When $\ell>2$, there are two different programs for constructing $M$. Both start by using the norm residue symbol of $\underline{a}$ to construct a nonzero element $\mu$ of $H^{2 b+1, b}(\mathfrak{X})$. The construction of $\mu$ is given in $[\mathrm{MC} / 2$, p. 95] and [MC/1, (5.2)] (see 2.5 below).

In Voevodsky's approach, the triangles (0.4) and (0.5) are constructed in (5.5) and (5.6) of $[\mathrm{MC} / \mathrm{l}]$ with $M=M_{\ell-1}$ and $D=M_{\ell-2}$, starting with $\mu \in H^{2 b+1, b}(\mathfrak{X})$. The duality axiom $0.3(\mathrm{~b})$ for $M$ and $D$ is given by $[\mathrm{MC} / 1,5.7]$.

Unfortunately, there is a gap in the proof of Lemma [MC/1, 5.15], which asserts that Axiom 0.3(a) holds, i. e., that $M_{\ell-1}$ is a summand of $X$. That proof depends via Theorems 3.8 and 4.4 of [MC/l] upon Lemmas 2.2 and 2.3 of [MC/l], whose proofs are currently unavailable. Instead, $[\mathrm{MC} / 1,5.11]$ proves that the canonical map $X \rightarrow \mathfrak{X}$ factors through the map $y: M \rightarrow \mathfrak{X}$.

In Rost's approach, $\mu$ defines an equivalence class of idempotent endomorphisms $e$ of $X$ and Rost defines $M$ to be the Chow motive $(X, e)$. By construction, his $M$ satisfies Axioms 0.3(a-b). Hopefully it also satisfies Axiom 0.3(c), the existence of triangles $(0.4)$ and $(0.5)$.

Notation The integer $n$ and the prime $\ell>2$ will be fixed. We will work over a fixed field $k$ in which $\ell$ is invertible. The integer $d$ will always be $\ell^{n-1}-1$ and $b$ will always be $d /(\ell-1)=1+\cdots+\ell^{n-2}$.

We fix the sequence of units $\underline{a}=\left(a_{1}, \ldots, a_{n}\right)$, and $X$ will always denote a $d$ dimensional Rost variety relative to $\underline{a}$, satisfying Axioms 0.3 .

We will work in the triangulated category of motives $\mathbf{D} \mathbf{M}^{\text {eff }}$ described in [MVW]. The Lefschetz motive is $\mathbb{L}=\mathbb{Z}(1)[2]$. Unless explicitly stated otherwise, motivic cohomology will always be taken with coefficients $\mathbb{Z}_{(\ell)}$. The notation $H_{\text {ét }}^{p, q}(-)$ refers to the étale motivic cohomology $H_{\text {ét }}^{p}\left(-, \mathbb{Z}_{(\ell)}(q)\right)$ defined in [MVW, 10.1].

Acknowledgements. This paper is an attempt to clarify Voevodsky's preprint [MC/l]. It is directly inspired by the work of Markus Rost, especially [2], who explicitly suggested the line of attack, based upon Axiom 0.3(c), which is presented in this paper. I am greatly indebted to both of them.

Finally, I would like to thank the Institute for Advanced Study for allowing me to present these results in a seminar during the Fall of 2006, and Pierre Deligne for his continued interest and encouragement. 


\section{Proof of Theorem 0.1}

We begin by giving the definition of a Rost variety. Recall $[4,1.20]$ that a $\nu_{n-1}$ variety over a field $k$ is a smooth projective variety $X$ of dimension $d=\ell^{n-1}-1$, with $\operatorname{deg} s_{d}(X) \not \equiv 0\left(\bmod \ell^{2}\right)$. Here $s_{d}(X)$ is the characteristic class of the tangent bundle $T_{X}$ corresponding to the symmetric polynomial $\sum t_{j}^{d}$ in the Chern roots $t_{j}$ of $T_{X}$; see [RPO, 14.3].

def:Rostvar Definition 1.1. A Rost variety for a sequence $\underline{a}=\left(a_{1}, \ldots, a_{n}\right)$ of units in $k$ is a $\nu_{n-1}$-variety such that: $\left\{a_{1}, \ldots, a_{n}\right\}$ vanishes in $\bar{K}_{n}^{M}(k(X)) / \ell$; for each $i<n$ there is a $\nu_{i}$-variety mapping to $X$; and the motivic homology sequence

$$
H_{-1,-1}\left(X^{2}\right) \stackrel{\pi_{0}^{*}-\pi_{1}^{*}}{\longrightarrow} H_{-1,-1}(X) \rightarrow H_{-1,-1}(k) \quad\left(=k^{\times}\right) .
$$

is exact. As mentioned in the Introduction, Rost varieties exist for every $\underline{a}$ by [4].

Example 1.2.1. If $n=1$ and $L=k\left(\sqrt[\ell]{a_{1}}\right)$, then $\operatorname{Spec}(L)$ is a Rost variety for $a_{1}$, with the convention that $s_{0}(X)=[L: k]=\ell$. When $n=2$, the Severi-Brauer variety corresponding to the degree $\ell$ algebra with symbol $\underline{a}$ is a Rost variety.

Proof of Theorem 0.1. . By [MC/2, 6.10], it suffices to prove the assertion

$$
H 90(n, \ell): H_{\text {ét }}^{n+1, n}(k)=0 .
$$

As pointed out in the proof of $[\mathrm{MC} / 2,7.4]^{1}$, it suffices to show that for every symbol $\underline{a} \in K_{n}^{M}(k)$ there is a field extension $k \subset F$ such that $\underline{a}$ vanishes in $K_{n}^{M}(F) / \ell$ and $H_{\text {ét }}^{n+1, n}(k) \rightarrow H_{\text {ét }}^{n+1, n}(F)$ is an injection. When $F=k(X)$, this is the bottom right arrow in the flowchart (1.3), which is implicit in [MC/l, 6.11].

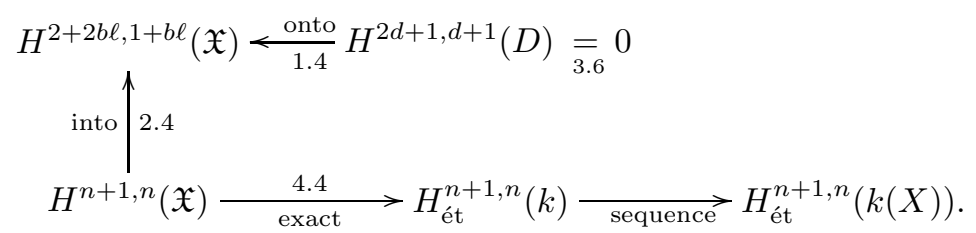

The other decorations in (1.3) refer to the properties we need and where they are established. Proposition 4.4 states that the bottom row of the flowchart (1.3) is exact. By 2.4 the group $H^{n+1, n}(\mathfrak{X})$ injects (by a cohomology operation) into $H^{2+2 b \ell, 1+b \ell}(\mathfrak{X})$, which is in turn a quotient of $H^{2 d+1, d+1}(D)$ by 1.4 . In 3.6, we prove that $H^{2 d+1, d+1}(D)=0$. All of this implies that $H_{\text {ét }}^{n+1, n}(k) \rightarrow H_{\text {ét }}^{n+1, n}(k(X))$ is an injection, finishing the proof of Theorem 0.1.

One part of the flowchart (1.3) is easy to establish.

6.13 Lemma 1.4. The map $s: \mathfrak{X} \rightarrow D \otimes \mathbb{L}^{b}[1]$ in (0.4) induces a surjection:

$$
H^{2 d+1, d+1}(D) \cong H^{2 b \ell+2, b \ell+1}\left(D \otimes \mathbb{L}^{b}[1]\right) \stackrel{s}{\longrightarrow} H^{2 b \ell+2, b \ell+1}(\mathfrak{X}) .
$$

Proof. In the cohomology exact sequence arising from (0.4), the next term is $H^{2 b \ell+2, b \ell+1}(M)$, which is a summand of $H^{2 b \ell+2, b \ell+1}(X)$. Because $b \ell=d+b>d$, this group is zero by the Vanishing Theorem [MVW, 3.6] — which says that $H^{n, i}(X)=0$ whenever $n>i+\operatorname{dim}(X)$.

\footnotetext{
$1_{\text {also known as }[6,7.1]}$
} 
The lower left map in flowchart (1.3) is just the motivic-to-étale map by the following basic result, which we quote from $[\mathrm{MC} / 2,7.3]^{2}$

7.3 Lemma 1.5. The structure map $\mathfrak{X} \rightarrow \operatorname{Spec}(k)$ induces isomorphisms

$$
H_{e ́ t}^{*, *}(k) \cong H_{e ́ t}^{*, *}(\mathfrak{X}) \quad \text { and } \quad H_{e ́ t}^{*, *}(k ; \mathbb{Z} / \ell) \cong H_{e ́ t}^{*, *}(\mathfrak{X} ; \mathbb{Z} / \ell) .
$$

\section{Motivic Cohomology Operations}

In the course of the proof, we will need some facts about the motivic cohomology operations constructed in [RPO]. When $\ell>2$, there are operations $P^{i}$ on $H^{*, *}(Y ; \mathbb{Z} / \ell)$ of bidegree $(2 i(\ell-1), i(\ell-1))$, for each $i \geq 0$ (with $P^{0}=1$ ), as well as the Bockstein operation $\beta$ of bidegree $(1,0)$. These satisfy the Adem relations given in [3] for the usual topological cohomology operations. In fact, the subring of all (stable) motivic cohomology operations generated by the $P^{i}$ and $\beta$ is isomorphic to the topologists' Steenrod algebra $\mathcal{A}^{*}$, developed in [3].

In addition, the motivic operations satisfy the usual Cartan formula $P^{n}(x y)=$ $\sum P^{i}(x) P^{j}(y), P^{i}(x)=x^{\ell}$ if $x \in H^{2 i, i}(Y ; \mathbb{Z} / \ell)$, and $P^{i}=0$ on $H^{p, q}(Y)$ when $(p, q)$ is in the region $q \leq n, p<n+q$. These are proven in [RPO, 9.7-9].

Still assuming $\ell>2$, the dual to the usual Steenrod algebra $\mathcal{A}^{*}$ is a gradedcommutative algebra on generators $\xi_{i}$ in (even) degrees $\left(2 \ell^{i}-2, \ell^{i}-1\right)$ and $\tau_{i}$ in (odd) degrees $\left(2 \ell^{i}-1, \ell^{i}-1\right)$; see [RPO, 12.6]. The dual to $\tau_{i}$ is the motivic cohomology operation $Q_{i}$, which has bidegree $\left(2 \ell^{i}-1, \ell^{i}-1\right)$. Because it is true in $\mathcal{A}^{*}$, the $Q_{i}$ are derivations which form an exterior subalgebra of all (stable) motivic cohomology operations. They may be inductively defined by $Q_{0}=\beta$ and $Q_{i+1}=\left[P^{\ell^{i}}, Q_{i}\right]$.

We now quickly establish those portions of $[\mathrm{MC} / \mathrm{l}]$ that we need, concerning these operations on the cohomology of $\mathfrak{X}$ and the cone $\Sigma \mathfrak{X}$ of $\mathfrak{X} \rightarrow \operatorname{Spec}(k)$. The proofs we give are due to Voevodsky, and only depend upon [MC/2] and [RPO]. In particular, they do not depend upon the missing lemmas in op. cit., or upon the Axioms 0.3.

Fix $q<n$. Because we have assumed that the norm residue map is an isomorphism in weight $q$, and hence that $H 90(q, \ell)$ holds, it follows from 1.5 that $H^{p, q}(k ; \mathbb{Z} / \ell) \cong H_{\text {ét }}^{p, q}(k ; \mathbb{Z} / \ell) \cong H_{\text {ét }}^{p, q}(\mathfrak{X} ; \mathbb{Z} / \ell)$ for $p \leq q$, and that $H_{\text {ét }}^{q+1, q}(\mathfrak{X} ; \mathbb{Z} / \ell)=0$. As observed in $[\mathrm{MC} / 1,6.6]$, it then follows from $[\mathrm{MC} / 2,6.9]^{3}$ that

$$
H^{p, q}(\Sigma \mathfrak{X} ; \mathbb{Z} / \ell)=0 \text { when }(p, q) \text { is in the region } q<n, p \leq 1+q .
$$

Since $X$ is a $\nu_{\leq n-1}$-variety, Theorem $[\mathrm{MC} / 2,3.2]$ translates to:

4.3 Theorem 2.2. If $i<n$, the sequences $\stackrel{Q_{i}}{\longrightarrow} H^{*, *}(\Sigma \mathfrak{X} ; \mathbb{Z} / \ell) \stackrel{Q_{i}}{\longrightarrow}$ are exact.

Remark 2.2.1. A slight generalization is stated in [MC/1, 4.3].

ex:4.3 Example 2.2.2. For $(p, q)=(n-2 \ell+3, n-\ell+1)$ we have the exact sequence

$$
H^{p, q}(\Sigma \mathfrak{X} ; \mathbb{Z} / \ell) \stackrel{Q_{1}}{\longrightarrow} H^{n+2, n}(\Sigma \mathfrak{X} ; \mathbb{Z} / \ell) \stackrel{Q_{1}}{\longrightarrow} H^{n+2 \ell+1, n+\ell-1}(\Sigma \mathfrak{X} ; \mathbb{Z} / \ell) .
$$

Because $p \leq q \leq n$, the left group is zero by (2.1). Thus the right map $Q_{1}$ is an injection on $H^{n+2, n}(\Sigma \mathfrak{X} ; \mathbb{Z} / \ell)$.

SXexp Lemma 2.3. The motivic cohomology groups $H^{*, *}(\Sigma \mathfrak{X})$ have exponent $\ell$.

\footnotetext{
2 alias [6, 7.2].

3 alias [6, 6.7]
} 
Proof. We may assume, by the usual transfer argument, that $k$ has no extensions of degree prime to $\ell$. In this case, the variety $X$ has a $k^{\prime}$-point for the field $k^{\prime}=k\left(\sqrt[\ell]{a_{1}}\right)$ of degree $\ell$ over $k$, by $[4,1.23]$. It follows that $\mathfrak{X} \otimes \operatorname{Spec} k^{\prime} \cong M\left(\operatorname{Spec} k^{\prime}\right)$, and hence that $\Sigma \mathfrak{X} \otimes \operatorname{Spec}\left(k^{\prime}\right) \cong 0$ The usual transfer argument now gives the result.

For any $p>q$ we have $H^{p, q}(\operatorname{Spec} k)=0$ and hence $H^{p, q}(\mathfrak{X}) \cong H^{p+1, q}(\Sigma \mathfrak{X})$. It follows from 2.3 that $H^{p, q}(\mathfrak{X}) \rightarrow H^{p, q}(\mathfrak{X} ; \mathbb{Z} / \ell)$ is an injection. By $[\mathrm{MC} / 2,7.2]$, the cohomology operations $Q_{i}$ preserve integral classes, so they induce integral operations on $H^{p, q}(\mathfrak{X})$ in this range.

Consider the cohomology operation $Q=Q_{n-1} \cdots Q_{2} Q_{1}$.

6.12 Lemma 2.4. The operation $Q: H^{n+1, n}(\mathfrak{X} ; \mathbb{Z} / \ell) \rightarrow H^{2+2 b \ell, 1+b \ell}(\mathfrak{X} ; \mathbb{Z} / \ell)$ is an injection, and induces an injection

$$
H^{n+1, n}(\mathfrak{X}) \hookrightarrow H^{2+2 b \ell, 1+b \ell}(\mathfrak{X}) .
$$

Proof. (Voevodsky) By the above remarks, it suffices to show that the operation $Q$ from $H^{n+2, n}(\Sigma \mathfrak{X} ; \mathbb{Z} / \ell)$ to $H^{3+2 b \ell, 1+b \ell}(\Sigma \mathfrak{X} ; \mathbb{Z} / \ell)$ is injective. As illustrated in Example 2.2.2, it is easy to see from 2.2 and (2.1) that each $Q_{i}$ is injective on the group $H^{*, *}(\Sigma \mathfrak{X} ; \mathbb{Z} / \ell)$ containing $Q_{i-1} \cdots Q_{1} H^{n+2, n}(\Sigma \mathfrak{X} ; \mathbb{Z} / \ell)$, because the preceding term in 2.2 is zero.

6.7 Remark 2.5. The same argument, given in [MC/1, 6.7], shows that $Q^{\prime}=Q_{n-2} \cdots Q_{0}$ is an injection from $H^{n, n-1}(\mathfrak{X} ; \mathbb{Z} / \ell)$ to $H^{2 b+1, b}(\mathfrak{X}) \subset H^{2 b+1, b}(\mathfrak{X} ; \mathbb{Z} / \ell)$.

If $\left\{a_{1}, \ldots, a_{n}\right\} \neq 0$ in $K_{n}^{M}(k) / \ell$, Voevodsky shows in [MC/l, 6.5] that its norm residue symbol in $H_{\text {ét }}^{n}\left(k, \mu_{\ell}^{\otimes n}\right)$ lifts to a nonzero element $\delta \in H^{n, n-1}(\mathfrak{X} ; \mathbb{Z} / \ell)$. Using injectivity of $Q^{\prime}$, we get a nonzero symbol $\mu=Q^{\prime}(\delta) \in H^{2 b+1, b}(\mathfrak{X})$. This symbol is the starting point of the construction of the motive $M$ in both the program of Voevodsky [MC/1] and that of Rost [2].

\section{Motivic Homology}

In this section, we prove Corollary 3.6, which depends upon Axiom 0.3(b) and exactness of (1.2) via results about the motivic homology of $\mathfrak{X}$ and $M$.

We will make repeated use of the following basic lemma. In this section, that the notation $H_{-p,-q}(Y)$ refers to the group $\operatorname{Hom}(\mathbb{Z}, Y(q)[p])$; see [MVW, 14.17].

Hombound Lemma 3.1. For every smooth (simplicial) $Y$ and $p>q, \operatorname{Hom}(\mathbb{Z}, Y(q)[p])=0$.

Proof. By [MVW, 14.16], $\operatorname{Hom}(\mathbb{Z}, Y(q)[p]) \cong H_{\mathrm{zar}}^{p-q}\left(k, C_{*}\left(Y \times \mathbb{G}_{m}^{q}\right)\right)=H^{p-q} C_{*}(Y \times$ $\left.\mathbb{G}_{m}^{q}\right)(k)$. The chain complex $C_{*}\left(Y \times \mathbb{G}_{m}^{q}\right)$ is zero in positive cohomological degrees, so the $H^{p-q}$ group vanishes.

6.15x Lemma 3.2. The structural map $H_{-1,-1}(\mathfrak{X}) \rightarrow H_{-1,-1}(k)=k^{\times}$is injective.

Proof. (Voevodsky) By Lemma 3.1, $\operatorname{Hom}\left(\mathbb{Z}, X^{p}(1)[n]\right)=0$ for all $n \geq 2$ and all $p$. Therefore the fourth quadrant spectral sequence

$$
E_{p, q}^{1}=\operatorname{Hom}\left(\mathbb{Z}, X^{p+1}(1)[-q]\right) \Rightarrow \operatorname{Hom}(\mathbb{Z}, \mathfrak{X}(1)[p-q])
$$

has only two nonzero rows $(q=0,-1)$. The row $q=-1$ yields the exact sequence

$$
0 \leftarrow H_{-1,-1}(\mathfrak{X}) \leftarrow H_{-1,-1}(X) \leftarrow H_{-1,-1}(X \times X) .
$$

Since (1.2) is exact, this implies the result.

6.15 Corollary 3.3. The structural map $H_{-1,-1}(M) \stackrel{y}{\longrightarrow} H_{-1,-1}(k)=k^{\times}$is injective. 
Proof. By $(0.4)$ and 3.2, it suffices to show that $\operatorname{Hom}(\mathbb{Z}, D(b+1)[2 b+1])=0$. By $(0.5)$ this follows from the vanishing of $\operatorname{Hom}(\mathbb{Z}, M(b+1)[2 b+1])$ and $\operatorname{Hom}(\mathbb{Z}, \mathfrak{X} \otimes$ $\left.\mathbb{L}^{b+d+1}\right)$, and both of these vanish by Lemma 3.1.

weight1 Lemma 3.4. $H^{0,1}(\mathfrak{X})=H^{2,0}(\mathfrak{X})=0$ and $H^{1,1}(\mathfrak{X} ; \mathbb{Z}) \cong H^{1,1}(\operatorname{Spec} k ; \mathbb{Z}) \cong k^{\times}$.

Proof. The spectral sequence $E_{1}^{p, q}=H^{q, 1}\left(X^{p+1} ; \mathbb{Z}\right) \Rightarrow H^{p+q}(\mathfrak{X} ; Z)$ degenerates, all rows vanishing except for $q=1$ and $q=2$, because $\mathbb{Z}(1) \cong \mathcal{O}^{\times}[-1]$; see [MVW, 4.2]. Hence for $q \leq 2$ we have $H^{q, 1}(\mathfrak{X})=H_{\text {ét }}^{q, 1}(\mathfrak{X})=H_{\text {ét }}^{q, 1}(k)=H_{\text {ét }}^{q-1}\left(k, \mathbb{G}_{m}\right)$.

6.14dual Lemma 3.5. $H^{2 d+1, d+1}(D ; \mathbb{Z})$ is the kernel of $H_{-1,-1}(M) \stackrel{y}{\longrightarrow} H_{-1,-1}(k)=k^{\times}$.

Proof. From (0.5) we get an exact sequence with coefficients $\mathbb{Z}$ :

$$
H^{2 d, d+1}\left(\mathfrak{X} \otimes \mathbb{L}^{d}\right) \rightarrow H^{2 d+1, d+1}(D) \rightarrow H^{2 d+1, d+1}(M) \stackrel{D y}{\longrightarrow} H^{2 d+1, d+1}\left(\mathfrak{X} \otimes \mathbb{L}^{d}\right) .
$$

The first group is $H^{0,1}(\mathfrak{X})$, which is zero by 3.4 , so it suffices to show that the map $D y$ identifies with the structural map $y$. This follows from Axiom 0.3(b), because for any $u$ in $H_{-1,-1}(M)=\operatorname{Hom}(\mathbb{Z}, M(1)[1])$ the $\mathfrak{X}$-dual of $\mathbb{Z}(-1)[-1] \stackrel{u}{\longrightarrow} M \stackrel{y}{\longrightarrow} \mathbb{Z}$ is

$$
\mathfrak{X} \otimes \mathbb{L}^{d} \rightarrow \mathbb{L}^{d} \stackrel{y^{*}}{\longrightarrow} M^{*} \otimes \mathbb{L}^{d} \stackrel{u^{*}}{\longrightarrow} \mathbb{Z}(1)[1] \otimes \mathbb{L}^{d}=\mathbb{Z}(d+1)[2 d+1] .
$$

6.14 Corollary 3.6. $H^{2 d+1, d+1}(D)=H^{2 d+1, d+1}(D ; \mathbb{Z}) \otimes \mathbb{Z}_{(\ell)}=0$.

\section{Between motivic and Étale Cohomology}

Definition 4.1. $\left([\mathrm{MC} / 2 \text {, p. } 90]^{4}\right)$ Let $L(n)$ denote the truncation $\tau^{\leq n+1} \mathbb{Z}_{(\ell)}^{\text {ét }}(n)$ of the complex in $\mathbf{D} \mathbf{M}^{\mathrm{eff}}$ representing étale motivic cohomology; i. e., $H^{p}(-, L(n)) \cong$ $H_{\text {ét }}^{p, n}(-)$ for $p \leq n+1$. Let $K(n)$ denote the cofiber of the canonical map $\mathbb{Z}_{(\ell)} \rightarrow$ $L(n)$.

6.10a Lemma 4.2. The map $H^{n+1}(\mathfrak{X}, K(n)) \stackrel{y}{\longrightarrow} H^{n+1}(M, K(n))$ is an injection.

Proof. By (0.4) we have an exact sequence:

$$
H^{n-1}\left(D \otimes \mathbb{L}^{b}, K(n)\right) \rightarrow H^{n}(\mathfrak{X}, K(n)) \stackrel{y}{\longrightarrow} H^{n}(M, K(n)) .
$$

We need to see that the left term vanishes. By (0.5), it suffices to show that $H^{n-1}\left(M \otimes \mathbb{L}^{b}, K(n)\right)$ and $H^{n-2}\left(\mathfrak{X} \otimes \mathbb{L}^{2 b}, K(n)\right)$ vanish. This follows from $[\mathrm{MC} / 2$, $6.12]^{5}$, which says that $H^{*}(Y(1), K(n))=0$ for every smooth $Y$, the assumption that $M$ is a summand of $X$, and the consequent collapsing of the spectral sequence $E_{1}^{p, q}=H^{q}\left(X^{p}, K(n)\right) \Rightarrow H^{p+q}(\mathfrak{X}, K(n))$.

6.10 Corollary 4.3. The map $H^{n+1}(\mathfrak{X}, K(n)) \rightarrow H^{n+1}(k(X), K(n))$ is an injection.

Proof. The map $H^{n+1}(X, K(n)) \rightarrow H^{n+1}(k(X), K(n))$ is an injection by [MVW, $11.1,13.8,13.10]$, or by $[\mathrm{MC} / 2,7.4]^{6}$. The corollary follows from 4.2 , since $H^{*}(M,-)$ is a summand of $H^{*}(X,-)$.

6.9 Proposition 4.4. There is an exact sequence

$$
H^{n+1, n}(\mathfrak{X}) \rightarrow H_{e ́ t}^{n+1, n}(k) \rightarrow H_{e ́ t}^{n+1, n}(k(X)) .
$$

\footnotetext{
4 alias $[6$, p. 32]

5 alias $[6,7.3]$

6 alias $[6,6.13]$
} 
Proof. By $1.5, \mathfrak{X} \rightarrow \operatorname{Spec}(k)$ is an isomorphism on étale motivic cohomology, so we have $H_{\text {ét }}^{n+1, n}(k) \cong H^{n+1}(\mathfrak{X}, L(n))$.

The map $\operatorname{Spec} k(X) \rightarrow \mathfrak{X}$ induces a commutative diagram with exact rows:

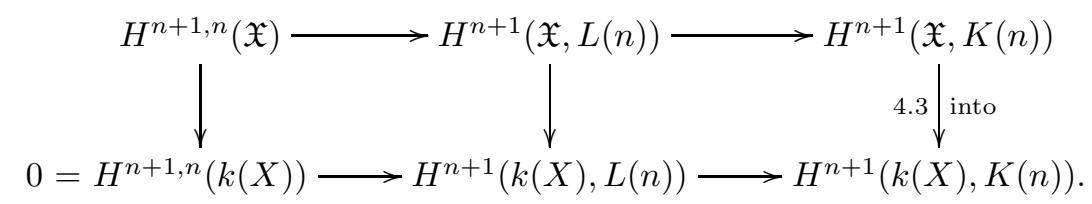

By 4.3, the right vertical map is an injection. The proposition now follows by a diagram chase.

\section{REFERENCES}

[MC/2] V. Voevodsky, Motivic Cohomology with $\mathbb{Z} / 2$ coefficients, Publ. IHES 98 (2003), 1-57.

[MC/l] V. Voevodsky, On Motivic Cohomology with Z/l coefficients, Preprint,2003. Available at http://www.math.uiuc. edu/K-theory/0639/.

[RPO] V. Voevodsky, Reduced Power operations in Motivic Cohomology, Publ. IHES 98 (2003), $1-57$.

[MVW] C. Mazza, V. Voevodsky and C. Weibel, Lecture notes on motivic cohomology, Clay Monographs in Math. 2, AMS, 2006.

[1] M. Rost, Chain lemma for splitting fields of symbols, Preprint, 1998. Available at http: //www.math. uni-bielefeld.de/rost/chain-lemma.html

[2] M. Rost, On the Basic Correspondence of a Splitting Variety, Preprint under construction, 2006.

[3] N. Steenrod and D. Epstein, Cohomology Operations, Annals of Math Studies 50, 1962.

[4] A. Suslin and S. Joukhovitsky, Norm Varieties, J. Pure Appl. Alg. 206 (2006), 245-276.

[5] A. Suslin and V. Voevodsky, Bloch-Kato conjecture and motivic cohomology with finite coefficients, pp. 117-189 in The arithmetic and geometry of algebraic cycles (Banff, 1998), NATO ASI Ser. C, vol. 548, Kluwer, Dordrecht, 2000.

[6] V. Voevodsky, Motivic Cohomology with $\mathbb{Z} / 2$ coefficients, Preprint. Available at http://www. math.uiuc. edu/K-theory/0502/, 2001.

[7] V. Voevodsky, Motives over simplicial schemes, Preprint. Available at http://www.math. uiuc. edu/K-theory/0638/, 2003.

Institute for Advanced Study, Princeton, NJ

Dept. of Mathematics, Rutgers University, New Brunswick, NJ 08901, USA

E-mail address: weibel@math.rutgers.edu 